\title{
Safeguard against DNA sensing: the role ofTREX1 in HIV-1 infection and autoimmune diseases
}

\author{
Maroof Hasan ${ }^{1,2}$ and Nan Yan ${ }^{1,2}$ * \\ ${ }^{1}$ Department of Internal Medicine, University of Texas Southwestern Medical Center, Dallas, TX, USA \\ ${ }^{2}$ Department of Microbiology, University of Texas Southwestern Medical Center, Dallas, TX, USA
}

\author{
Edited by: \\ Nadine Laguette, Centre National de \\ la Recherche Scientifique, France

\section{Reviewed by:} \\ Warner C. Greene, J. David Gladstone \\ Institutes, USA \\ Judy Lieberman, Boston Children's \\ Hospital, USA

\section{${ }^{*}$ Correspondence:} \\ Nan Yan, Department of Internal \\ Medicine and Department of \\ Microbiology, University of Texas \\ Southwestern Medical Center, Dallas, \\ TX 75390-9113, USA \\ e-mail: nan.yan@utsouthwestern.edu
}

Innate immune recognition is crucial for host responses against viral infections, including infection by human immunodeficiency virus 1 (HIV-1). Human cells detect such invading pathogens with a collection of pattern recognition receptors that activate the production of antiviral proteins, such as the cytokine interferon-type I, to initiate antiviral responses immediately as well as the adaptive immune response for long-term protection. To establish infection in the host, many viruses have thus evolved strategies for subversion of these mechanisms of innate immunity. For example, acute infection by HIV-1 and other retroviruses have long been thought to be non-immunogenic, signifying suppression of host defenses by these pathogens. Studies in the past few years have begun to uncover a multifaceted scheme of how HIV-1 evades innate immune detection, especially of its DNA, by exploiting host proteins. This review will discuss the host mechanisms of HIV-1 DNA sensing and viral immune evasion, with a particular focus on TREX1, three prime repair exonuclease 1, a host $3^{\prime}$ exonuclease (also known as DNase III).

Keywords: HIV, innate immunity, DNA sensing, Trex1, autoimmune diseases

\section{THE INTERFERON RESPONSE TO HIV DNA}

Human immunodeficiency virus (HIV) enters $\mathrm{T}$ cells and macrophages by first interacting with host receptor CD4 then with co-receptor chemokine (C-C motif) receptor 5 (CCR5) or chemokine (C-X-C motif) receptor 4 (CXCR4) on the target cell plasma membrane, triggering viral envelope fusion. HIV can also bind to cell surface lectins (sugar-binding proteins) and enter cells by endocytosis. This is the predominant mode of entry into dendritic cells (DCs), which play important roles in progression and pathology of HIV infection (Luban, 2012; Manches et al., 2014), although HIV-1 does not replicate efficiently in DCs. Regardless of the mode of viral entry, the viral core containing its RNA is released into the cytosol, and HIV-encoded reverse transcriptase begins to convert viral RNA into DNA while still encapsulated in the capsid core. After completion of reverse transcription, viral integrase binds to both ends of full-length HIV-1 DNA to form pre-integration complex, which delivers functional HIV DNA to the host nucleus for chromosomal integration (Goff, 2007). Since only a few copies of HIV DNA integrate, the bulk of HIV DNA remains in the cytosol unless cleared by host enzymes (Yan et al., 2010).

Although abundant, the HIV-encoded cytosolic DNA produced by reverse transcription does not trigger a cell-autonomous interferon (IFN) or inflammatory response in activated CD4 T cells and macrophages, its primary targets (Goldfeld et al., 1991; Yan et al., 2010). HIV achieves immune evasion in these target cells by exploiting the host DNase TREX1 (Yan et al., 2010), the most abundant exonuclease in mammalian cell (Hoss et al., 1999; Mazur and Perrino, 1999), to clear its DNA. This action of TREX1 diminishes HIV DNA products in the cytosol below the threshold of immune activation. This is strikingly demonstrated in
Trex1-/- or knockdown cells. In the absence of TREX1, HIV infection triggers a robust type I IFN response strictly dependent upon the cytosolic DNA sensing pathway, including the endoplasmic reticulum (ER) localized adaptor stimulator of interferon genes (STING), TANK-binding kinase 1 (TBK1), and the transcription factor interferon regulatory factor 3 (IRF3) (Yan et al., 2010). The HIV-stimulated IFN response in Trex $1-/-$ cells can be dampened by reverse transcriptase inhibitor (e.g., nevirapine) but not by integrase inhibitor (e.g., raltegravir), consistent with HIV DNA being the main pathogen associated molecular pattern (PAMP) detected by an innate immune sensor (Yan et al., 2010). The HIV DNA is sensed by binding to cGAMP synthase (cGAS), which then synthesizes the unique second messenger dinucleotide cyclic GMP-AMP (cGAMP) that binds to STING to activate downstream IFN signaling (Gao et al., 2013; Figure 1A). This "competition" between TREX1 (proviral) and cGAS (antiviral) for cytosolic DNA also applies to murine leukemia virus (MLV) and simian immunodeficiency virus (SIV; Gao et al., 2013), and likely many other retroviruses.

Thus, the absence of an immunogenic response to acute retroviral infection of target immune cells can be at least partially explained by TREX1, which is ubiquitously expressed and at high levels in immune cells (Pereira-Lopes et al., 2013), and may synergize with other host factors (see below). In one population study comparing cohorts of healthy control and HIV-positive individuals, TREX1 polymorphisms associated with susceptibility to HIV infection (Pontillo et al., 2013), although another study using different cohorts of patients failed to detect such an association (Sironi et al., 2012), suggesting that further investigation is needed before genetic linkage is established. From an evolutionary standpoint, it is intriguing that TREX1 is only found in mammals that 


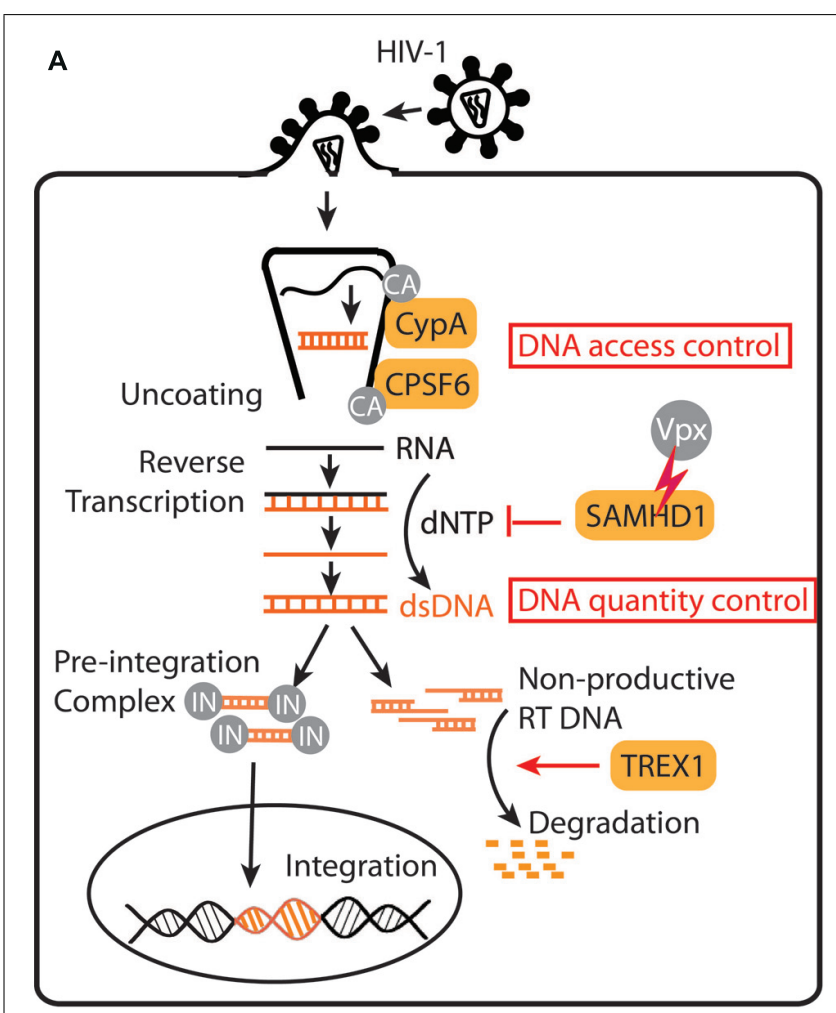

B

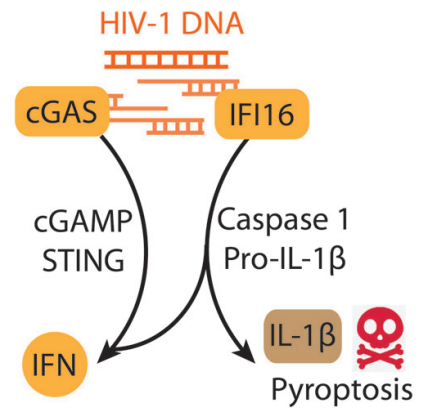

FIGURE 1 | HIV-1 subversion of innate immune DNA sensing. (A) A schematic diagram of early stages of HIV-1 life cycle. HIV-1 capsid (CA) and associated host factors, CypA and CPSF6, regulate HIV DNA access to innate immune sensing. TREX1 and SAMHD1 regulate HIV DNA quantity. IN, integrase. (B) HIV-1 DNA sensing by cGAS in many target cells activates a STING-mediated IFN response, whereas HIV-1 DNA sensing by IFI16 in "bystander" quiescent CD4+ T cells trigger IFN signaling, caspase-1 activation, IL-1 $\beta$ secretion and pyroptosis.

have co-evolved with retrovirus, suggesting that retroviruses have adapted to exploit TREX1 for survival. Such an adaptation may be particularly essential for HIV, which does not appear to encode its own factors to antagonize intracellular innate immune sensing systems like many other DNA and RNA viruses do. Indeed, these findings have engendered a new paradigm for HIV-host interactions - that HIV not only exploits many host factors for the successful completion of the life cycle (Brass et al., 2008; König et al., 2008), it also exploits several key host factors that are critical for subversion of innate immune responses in target cells (Doitsh et al., 2010, 2014; Manel et al., 2010; Yan et al., 2010).

\section{PROINFLAMMATORY RESPONSE TO HIV DNA}

HIV DNA can also trigger a proinflammatory response in nonproductively infected "bystander" CD4+ T cells and promote T cell killing (Doitsh et al., 2010). HIV replication is restricted in these "bystander" CD4+ T cells due to the action of SAM domain and HD domain containing protein 1 (SAMHD1) that depletes the dNTP pool, as well as other unknown restrictive factors (Baldauf et al., 2012). As a result, HIV replication in these cells arrests early in the reverse transcription stage, although the limited amount of DNA produced can be recognized by another cytosolic DNA sensor interferon-induciable protein 16 (IFI16) (Monroe et al., 2014; Figure 1B). IFI16 was initially identified as a sensor that recognizes viral DNA or exogenous double-stranded DNA (introduced by transfection) and signals via STING to activate the IFN response (Unterholzner et al., 2010). A recent study found that "bystander" CD4+ T cells harboring abortive HIV DNA products trigger IFI16-mediated IFN signaling and inflammasome response, including activation of caspase- 1 , secretion of IL- $1 \beta$, and death of the host cell by pyroptosis (Doitsh et al., 2014). This series of discoveries reveals another exciting example of how HIV takes advantage of DNA sensing as well as SAMHD1 restriction. In this case, instead of avoiding DNA sensing, HIV stalls DNA replication early in the reverse transcription stage to trigger inflammation and cell death in "bystander" CD4+ T cells. Since CD4+ T cell depletion is a highly diagnostic clinical feature of AIDS, these studies raise an exciting possibility of reversing $\mathrm{CD} 4+\mathrm{T}$ cell depletion by blocking the inflammasome response with caspase-1 inhibitors (Doitsh et al., 2014). Both cGAS and IFI16 sense HIV DNA, yet they seem to function in distinct cell types and lead to different consequences. Also, it was unclear why TREX1 is not able to inhibit IFI16-mediated detection of HIV DNA. Further investigation is needed to show what determines which innate immune signaling pathway HIV DNA triggers or avoids, and how that influences the overall fitness of the virus.

\section{QUANTITY CONTROL OF HIV DNA: TREX1 AND SAMHD1}

As discussed above, the amount of HIV-1 DNA in the cytosol depends upon the rates of synthesis and degradation by two host factors (Figure 1A): TREX1 mediates HIV DNA degradation in several immune cell types, and SAMHD1 limits HIV-1 DNA synthesis by depleting the dNTP pool in resting CD4+ $\mathrm{T}$ cells as well as several other cell types of myeloid linage (Hrecka et al., 2011; Laguette et al., 2011; Baldauf et al., 2012). In DCs, SAMHD1 also prevents innate immune activation (Manel et al., 2010; Sunseri etal., 2011). SAMHD1 restriction can be overcome by treating cells with virus-like particles (VLPs) containing the SAMHD1-antagnist protein Vpx, found in SIVmac and HIV-2 viruses (Goujon etal., 2006). HIV-1, which does not encode Vpx, Vpx-deficient SIVmac, and HIV-2 all fail to replicate efficiently in DCs (Hrecka et al., 2011; Laguette et al., 2011). Detailed reviews on SAMHD1/Vpx can be found elsewhere (Daugherty and Malik, 2012; Luban, 2012; Sze et al., 2013). Here, we highlight features of SAMHD1 as in comparison with TREX1.

TREX1 is expressed in most mammalian cell types (PereiraLopes et al., 2013), whereas SAMHD1 expression is more restricted (Laguette et al., 2011). Since a defect in either enzyme triggers 
HIV-1 DNA sensing, they are thought to play non-redundant roles. In cell culture, TREX1 deficiency leads to accumulation of total HIV-1 DNA, but not integrated proviral DNA (Yan et al., 2010). It therefore appears that TREX1 does not degrade fulllength integration-competent HIV-1 DNA, perhaps because it is protected by integrase in the pre-integration complex. In contrast, TREX1 appears able to degrade non-productive partial-length DNA generated by error-prone reverse transcription that are not incorporated into integrase complexes. This is consistent with enzymatic properties of TREX1, a $3^{\prime}$ to $5^{\prime}$ exonuclease. Although it has some activity toward any form of DNA, it is most efficient with single-stranded DNA or double-stranded DNA with a single-stranded overhang (Mazur and Perrino, 1999). Consequently, overall HIV-1 replication in which TREX1 expression is suppressed or ablated is reduced compared replication in normal cells, because DNA sensing results in expression of antiviral IFNs (Yan et al., 2010). In contrast, SAMHD1 depletion results in increased HIV DNA synthesis, integration and overall replication (Hrecka et al., 2011; Laguette et al., 2011). It appears confusing at first glance that although both TREX1 and SAMHD1 control the quantity of cytosolic HIV DNA, the overall outcome of HIV-1 replication is quite the opposite. Also, both proteins attenuate autoimmunity, and patients carrying defective TREX1 or SAMHD1 develop similar autoimmune and sterile inflammatory phenotypes (Crow et al., 2006; Crow and Rehwinkel, 2009; Rice et al., 2009). Therefore, important questions remain. Is the antiviral role of SAMHD1 in HIV-1 pathogenesis in the complex human immune setting different from current understanding of its role as a restrictive factor? Has HIV-1 evolved to exploit SAMHD1 restriction to prevent replication in certain cell types, such as DCs, in order to avoid cell-autonomous innate immune activation, or to abort the reverse transcription stage in "bystander" CD4+ T cells to activate inflammasomes and trigger pyroptosis?

Comparison of HIV-1 with HIV-2 offers some insights into these questions. HIV-2 (containing $\mathrm{Vpx}$ ) is far less pathogenic compared to HIV-1 (lacking Vpx) in humans, so one possible contributing factor to $\mathrm{HIV}-1$ pathogenesis could be its inability to antagonize SAMHD1 or ability to avoid SAMHD1 expressing cells. One should also consider other important differences between HIV-1 and HIV-2, such as Vpu, which is only encoded by HIV1, that antagonizes restriction factor Tetherin (Neil et al., 2008). Taken together, the opposing influences of TREX1 and SAMHD1 upon overall HIV-1 fitness suggests that it will be important to further evaluate these immunomodulators in combination as well as in isolation in immune systems.

\section{ACCESS CONTROL OF HIV DNA: CAPSID AND ASSOCIATED HOST FACTORS}

Aside from quantity, another factor in HIV-1 DNA sensing is encapsulation of the DNA by the viral capsid, which encapsulates the incoming genomic RNA and, later on, the reverse transcribed DNA. It remains unclear whether or not the HIV-1 capsid core disassembles during or after reverse transcription, and where in the cell it occurs. In any case, capsid core uncoating and reverse transcription are closely related. Several capsid assembly mutants affect reverse transcription (Tang et al., 2001; Ambrose et al., 2012). Conversely, inhibition of reverse transcription increases stability of the HIV-1 capsid core (Yang et al., 2013). Therefore, stability of the capsid core may play a role in HIV-1 DNA access or sensing. In the case of MLV, the glycosylated Gag protein (glyco-Gag) from the incoming virus enhances viral core stability and reduces DNA sensing in TREX1 knock-down cells, so a similar relationship may apply for HIV-1 (Stavrou et al., 2013). Moreover, host cofactor proteins that bind the capsid also modulate sensing of cytosolic HIV DNA. In one study (Lahaye et al., 2013), mutations of HIV1 and HIV-2 capsid proteins that enhance cyclophilin A (CypA) binding triggered more robust DNA sensing in monocyte-derived dendritic cells (MDDCs). In another study (Rasaiyaah et al., 2013), mutations N74D and P90A in the HIV-1 capsid protein impaired interaction with host factors cofactors cleavage and polyadenylation specificity factor sub-unit 6 (CPSF6) and CypA, and also promoted more DNA sensing in monocyte-derived macrophages (MDMs). Although different cell types were examined, both studies revealed an unexpected new dimension in sensing of HIV DNA by the host - access to the viral DNA dependent upon the viral capsid and factors encoded by the host. It remains to be determined whether the incoming capsid core simply "shields" HIV DNA from cytosolic sensing machinery by physical or steric means, or whether a more complex mechanism might be in play that regulates the dynamic connections of uncoating, reverse transcription, and nuclear import.

\section{SELF-DNA SENSING AND AUTOIMMUNE DISEASE}

Remarkable similarity in clinical conditions between infectious and autoimmune diseases has been recognized for many years. And yet, molecular mechanisms are much better defined in infectious diseases, whereas cell-intrinsic causes of autoimmune disease remain largely mysterious. TREX1 is an excellent example of a protein that may bridge this gap in our knowledge by playing important roles in both infectious and autoimmune diseases. In addition to its role in innate immunity against HIV discussed above, TREX1 is also a critical suppressor of self-recognition that safeguards the host from erroneous autoimmune activation. Mutations in TREX1 in humans are associated with the autoimmune and autoinflammatory disorders (Kavanagh et al., 2008; Crow and Rehwinkel, 2009) Aicardi-Goutières syndrome (AGS), familial chilblain lupus (FCL), systemic lupus erythematosus (SLE), and retinal vasculopathy with cerebral leukodystrophy (RVCL). In fact, TREX1 represents one of the highest monogenic linkages of SLE (Moser et al., 2009). Trex1-/- mice develop multiple-organ inflammation, as well as autoantibodies, and succumb to inflammation burden early in age (Gall et al., 2012). Self-DNA from endogenous retroelements (Stetson et al., 2008) or DNA replication debris (Yang et al., 2007) are among the likely causes of inflammation in Trex1-/- mice. Inflammatory phenotypes can be rescued by eliminating essential components of the known DNA sensing pathway (e.g., Irf3-/-, Sting-/-), functionally linking the DNase function of TREX1 to disease (Stetson et al., 2008; Gall et al., 2012). Moreover, chemically modified self-DNA that resists degradation by TREX1 can cause immune activation even in the presence of TREX1. One example is oxidized DNA that contains 8-hydroxyguanosin $(8-\mathrm{OHG})$, which can be formed in UV-exposed skin lesions or neutrophil extracellular traps (networks of extracellular fibers composed of mostly DNA from 
neutrophils). The oxidized DNA elevates cGAS/STING-mediated immune activation compared to unmodified DNA in a variety of cell types (Gehrke et al., 2013).

\section{TREX1: BEYOND DNase FUNCTION}

TREX1 mutations that disrupt its DNase activity were mostly found in AGS, and many disease-associated mutations of TREX1 do not affect its DNase activity, especially the ones associated with SLE and RVCL (Lee-Kirsch et al., 2007; Richards et al., 2007). TREX1 is a single exon gene that encodes an exonuclease domain at its amino terminus, and an ER localization domain at its carboxyl terminus. The ER localization domain consists of a hydrophilic linker region of unknown function [although this region harbors many SLE mutations (Crow and Rehwinkel, 2009)] and a small hydrophobic segment at the extreme carboxyl-terminus that sorts TREX1 to the cytosolic leaflet of the ER membrane by serving as an ER tail-anchor (rather than a classical transmembrane span; Lee-Kirsch et al., 2007; Lindahl et al., 2009). In overexpression studies, the C-terminal domain of TREX1 mediated interaction with ubiquilin-1, leading to monoubiquitination at multiple lysine residues of TREX1 (Orebaugh et al., 2013). These modifications did not lead to TREX1 degradation, rather, they are possibly able to regulate TREX1 function or localization (Hicke, 2001). Notably, several disease-causing mutations of TREX1 exhibit altered ubiquitination patterns when co-expressed with ubiquitin (Orebaugh et al., 2013), but do not affect DNase activity. Clearly, the biological significance of monoubiquitination on native or disease-causing forms of TREX1 for viral DNA sensing and autoimmunity requires further investigation.

Abnormal accumulation of self-DNA likely promotes TREX1 AGS, since these alleles impair DNase activity, but the molecular cause for TREX1 SLE that is strongly associated with mutations in the C-terminal localization domain rather than the DNase domain remains unclear. We recently found that TREX1-deficient cells have expanded lysosomal compartments, and that TREX1 deficiency promotes lysosomal biogenesis through mTORC1 and transcription factor TFEB (Hasan et al., 2013). It remains to be seen whether this new function of TREX1 is dependent upon its DNase activity, and how it relates to various autoimmune diseases associated with TREX1. Interestingly, although arising from the same genetic locus, clinical conditions of TREX1 AGS and TREX1 SLE are highly distinct. TREX1 AGS mutations are autosomal recessive. Patients develop severe neurological brain diseases with excess IFN $\alpha$ in cerebral spinal fluid resembling intrauterine infections, affecting mostly infant and young children, many of whom die early in age (disease onset usually by 4 months; Kavanagh et al., 2008). In contrast, TREX1 SLE mutations are mostly autosomal dominant, with two exceptions where compound heterozygous missense mutations were found (Lee-Kirsch et al., 2007). Unlike TREX1 AGS, which is a pediatric disorder, TREX1 SLE disease onset is usually between 15-40 years of age as is typical for lupustype autoimmune disorders. Similarly, TREX1 SLE patients have high titers of a wide array of autoantibodies and IFN signatures (Kavanagh etal., 2008). Despite some phenotypic overlap between SLE and AGS, distinct disease mechanisms are therefore likely.

\section{CONCLUSIONS AND PERSPECTIVES}

Extensive effort in the past two decades provided us with incredible details on HIV-1 virology, life cycle, interactions with host factors, and antagonism of host intrinsic restriction factors with accessory proteins (Goff, 2007). In more recent years, with the rapidly expanded understanding of innate immunity, studies have begun to uncover mechanistic details of how HIV affects host innate immunity (Luban, 2012). Like many other viruses, evasion of host innate immunity is one of the most essential requirements for overall fitness of HIV-1. However, what is remarkable about HIV-1 compared to other DNA or RNA viruses is how it achieves immune evasion by exploiting host proteins. Most DNA or RNA viruses encode viral proteins that target specific intracellular innate immune sensing pathways involved in recognizing DNA or RNA, or immune activation. HIV-1 does it by exploiting host negative regulators of innate immunity to subvert sensing of its own DNA. The focus of this review, DNase III/TREX1, normally safeguards the host from autoimmune activation by self-DNA, but is exploited by HIV-1 to evade sensing of its own DNA. In a seemingly counterintuitive move, $\mathrm{HIV}-1$ does not encode $\mathrm{Vpx}$ that antagonizes a potent restriction factor SAMHD1. Interestingly, $v p x$ deletion during the evolution of SIVcpz (that eventually gave rise to HIV-1) resulted in the creation of a unique vif that can antagonize hominid restriction factor APOBEC3 (Etienne et al., 2013). Whether this curious omission of Vpx contributes to overall HIV-1 fitness, and how much of that advantage is contributed from SAMHD1, remain to be seen. This could be an unprecedented way for a virus to subvert immune activation by keeping its replication low in professional IFN producing cells such as DCs. Along the same line, abortive HIV DNA products in bystander quiescent CD4+ T cells (due to the SAMHD1 block) activate IFI16-mediated inflammasome response and pyroptosis an effective way to paralyze the host immune system.

These discoveries of unique HIV immune evasion strategies may mark the beginning of an exciting new era on studies of HIV innate immunity, with important new avenues to be explored. Further studies in this emerging field will certainly open our eyes on how HIV navigates the human innate immune system. This knowledge could also be harnessed for novel strategies of HIV vaccine design that specifically target HIV immune evasion. We look forward to seeing these studies unfold.

\section{ACKNOWLEDGMENTS}

This work was supported by NIH AI98569 (Nan Yan). We thank Mark Lehrman for critical reading of the manuscript. We apologize for not being able to cite all related studies due to space limitation.

\section{REFERENCES}

Ambrose, Z., Lee, K., Ndjomou, J., Xu, H., Oztop, I., Matous, J., et al. (2012). Human immunodeficiency virus type 1 (HIV-1) capsid mutation N74D alters cyclophilin a dependence and impairs macrophage infection. J. Virol. 86, 4708-4714. doi: 10.1128/JVI.05887-11

Baldauf, H. M., Pan, X., Erikson, E., Schmidt, S., Daddacha, W., Burggraf, M., et al. (2012). SAMHD1 restricts HIV-1 infection in resting CD4 (+) T cells. Nat. Med. 18, 1682-1687. doi: 10.1038/nm.2964

Brass, A., Dykxhoorn, D., Benita, Y., Yan, N., Engelman, A., Xavier, R., et al. (2008). Identification of host proteins required for HIV infection through a functional genomic screen. Science 319, 921-926. doi: 10.1126/science.1152725 
Crow, Y., Hayward, B., Parmar, R., Robins, P., Leitch, A., Ali, M., et al. (2006). Mutations in the gene encoding the $3^{\prime}-5^{\prime}$ DNA exonuclease TREX1 cause Aicardi-Goutieres syndrome at the AGS1 locus. Nat. Genet. 38, 917-920. doi: $10.1038 / \mathrm{ng} 1845$

Crow, Y. J., and Rehwinkel, J. (2009). Aicardi-Goutieres syndrome and related phenotypes: linking nucleic acid metabolism with autoimmunity. Hum. Mol. Genet 18, R130-R136. doi: 10.1093/hmg/ddp293

Daugherty, M. D., and Malik, H. S. (2012). Rules of engagement: molecular insights from host-virus arms races. Annu. Rev. Genet. 46, 677-700. doi: 10.1146/annurevgenet-110711-155522

Doitsh, G., Cavrois, M., Lassen, K. G., Zepeda, O., Yang, Z., Santiago, M. L., et al. (2010). Abortive HIV infection mediates CD4 T cell depletion and inflammation in human lymphoid tissue. Cell 143, 789-801. doi: 10.1016/j.cell.2010.11.001

Doitsh, G., Galloway, N. L. K., Geng, X., Yang, Z., Monroe, K. M., Zepeda, O., et al (2014). Cell death by pyroptosis drives CD4 T-cell depletion in HIV-1 infection. Nature 505, 509-514. doi: 10.1038/nature12940

Etienne, L., Hahn, B. H., Sharp, P. M., Matsen, F. A., and Emerman, M. (2013). Gene loss and adaptation to hominids underlie the ancient origin of HIV-1. Cell Host Microbe 14, 85-92. doi: 10.1016/j.chom.2013.06.002

Gall, A., Treuting, P., Elkon, K. B., Loo, Y.-M., Gale, M., Barber, G. N., et al. (2012). Autoimmunity initiates in nonhematopoietic cells and progresses via lymphocytes in an interferon-dependent autoimmune disease. Immunity 36, 120-131. doi: 10.1016/j.immuni.2011.11.018

Gao, D., Wu, J., Wu, Y.-T., Du, F., Aroh, C., Yan, N., et al. (2013). Cyclic GMP-AMP synthase is an innate immune sensor of HIV and other retroviruses. Science 341 903-906. doi: 10.1126/science. 1240933

Gehrke, N., Mertens, C., Zillinger, T., Wenzel, J., Bald, T., Zahn, S., et al (2013). Oxidative damage of DNA confers resistance to cytosolic nuclease TREX1 degradation and potentiates STING-dependent immune sensing. Immunity 39, 482-495. doi: 10.1016/j.immuni.2013.08.004

Goff, S. (2007). Host factors exploited by retroviruses. Nat. Rev. Microbio. 5, 253 263. doi: $10.1038 /$ nrmicro 1541

Goldfeld, A. E., Birch-Limberger, K., Schooley, R. T., and Walker, B. D. (1991). HIV1 infection does not induce tumor necrosis factor-alpha or interferon-beta gene transcription. J. Acquir. Immune Defic. Syndr. 4, 41-47. doi: 10.1097/00126334199101000-00006

Goujon, C., Jarrosson-Wuillème, L., Bernaud, J., Rigal, D., Darlix, J.L., and Cimarelli, A. (2006). With a little help from a friend: increasing HIV transduction of monocyte-derived dendritic cells with virionlike particles of SIV (MAC). Gene Ther. 13, 991-994. doi: 10.1038/sj.gt. 3302753

Hasan, M., Koch, J., Rakheja, D., Pattnaik, A. K., Brugarolas, J., Dozmorov, I., et al. (2013). Trexl regulates lysosomal biogenesis and interferon-independent activation of antiviral genes. Nat. Immunol. 14, 61-71. doi: 10.1038/ni.2475

Hicke, L. (2001). Protein regulation by monoubiquitin. Nat. Rev. Mol. Cell Biol. 2, 195-201. doi: 10.1038/35056583

Hoss, M., Robins, P., Naven, T., Pappin, D., Sgouros, J., and Lindahl, T. (1999). A human DNA editing enzyme homologous to the Escherichia coli DnaQ/MutD protein. EMBO J. 18, 3868-3875. doi: 10.1093/emboj/18.13.3868

Hrecka, K., Hao, C., Gierszewska, M., Swanson, S. K., Kesik-Brodacka, M., Srivastava, S., etal. (2011). Vpx relieves inhibition of HIV-1 infection of macrophages mediated by the SAMHD1 protein. Nature 474, 658-661. doi: 10.1038 /nature10195

Kavanagh, D., Spitzer, D., Kothari, P. H., Shaikh, A., Liszewski, M. K., Richards, A., et al. (2008). New roles for the major human 3'-5' exonuclease TREX1 in human disease. Cell Cycle 7, 1718-1725. doi: 10.4161/cc.7.12.6162

König, R., Zhou, Y., Elleder, D., Diamond, T. L., Bonamy, G. M. C., Irelan, J. T., et al. (2008). Global analysis of host-pathogen interactions that regulate early-stage HIV-1 replication. Cell 135, 49-60. doi: 10.1016/j.cell.2008.07.032

Laguette, N., Sobhian, B., Casartelli, N., Ringeard, M., Chable-Bessia, C., Ségéral, E., et al. (2011). SAMHD1 is the dendritic- and myeloid-cell-specific HIV-1 restriction factor counteracted by Vpx. Nature 474, 654-657. doi: 10.1038 /nature 10117

Lahaye, X., Satoh, T., Gentili, M., Cerboni, S., Conrad, C., Hurbain, I., etal. (2013). The capsids of HIV-1 and HIV-2 determine immune detection of the viral cDNA by the innate sensor cGAS in dendritic cells. Immunity 39, 1132-1142. doi: 10.1016/j.immuni.2013. 11.002
Lee-Kirsch, M. A., Gong, M., Chowdhury, D., Senenko, L., Engel, K., Lee, Y.-A., et al. (2007). Mutations in the gene encoding the 3'-5' DNA exonuclease TREX1 are associated with systemic lupus erythematosus. Nat. Genet. 39, 1065-1067. doi: $10.1038 / \mathrm{ng} 2091$

Lindahl, T., Barnes, D. E., Yang, Y.-G., and Robins, P. (2009). Biochemical properties of mammalian TREX1 and its association with DNA replication and inherited inflammatory disease. Biochem. Soc. Trans. 37, 535-538. doi: 10.1042/BST0370535

Luban, J. (2012). Innate immune sensing of HIV-1 by dendritic cells. Cell Host Microbe 12, 408-418. doi: 10.1016/j.chom.2012.10.002

Manches, O., Frleta, D., and Bhardwaj, N. (2014). Dendritic cells in progression and pathology of HIV infection. Trends Immunol. 35, 114-122. doi: 10.1016/j.it.2013.10.003

Manel, N., Hogstad, B., Wang, Y., Levy, D. E., Unutmaz, D., and Littman, D. R. (2010). A cryptic sensor for HIV-1 activates antiviral innate immunity in dendritic cells. Nature 467, 214-217. doi: 10.1038/nature09337

Mazur, D., and Perrino, F. (1999). Identification and expression of the TREX1 and TREX2 cDNA sequences encoding mammalian $3^{\prime}->5^{\prime \prime}$ exonucleases. J. Biol. Chem. 274, 19655-19660. doi: 10.1074/jbc.274.28.19655

Monroe, K. M., Yang, Z., Johnson, J. R., Geng, X., Doitsh, G., Krogan, N. J., et al. (2014). IFI16 DNA sensor is required for death of lymphoid CD4 T cells abortively infected with HIV. Science 343, 428-432. doi: 10.1126/science.1243640

Moser, K. L., Kelly, J. A., Lessard, C. J., and Harley, J. B. (2009). Recent insights into the genetic basis of systemic lupus erythematosus. Genes Immun. 10, 373-379. doi: 10.1038/gene.2009.39

Neil, S., Zang, T., and Bieniasz, P. (2008). Tetherin inhibits retrovirus release and is antagonized by HIV-1 Vpu. Nature 451, 425-430. doi: 10.1038/nature06553

Orebaugh, C. D., Fye, J. M., Harvey, S., Hollis, T., Wilkinson, J. C., and Perrino, F. W. (2013). The TREX1 C-terminal region controls cellular localization through ubiquitination. J. Biol. Chem. 288, 28881-28892. doi: 10.1074/jbc.M113. 503391

Pereira-Lopes, S., Celhar, T., Sans-Fons, G., Serra, M., Fairhurst, A.-M., Lloberas, J., et al. (2013). The exonuclease Trexl restrains macrophage proinflammatory activation. J. Immunol. 191, 6128-6135. doi: 10.4049/jimmunol.1301603

Pontillo, A., Girardelli, M., Catamo, E., Duarte, A. J., and Crovella, S. (2013). Polymorphisms in TREX1 and susceptibility to HIV-1 infection. Int. J. Immunogenet. 40, 492-494. doi: 10.1111/iji.12071

Rasaiyaah, J., Tan, C. P., Fletcher, A. J., Price, A. J., Blondeau, C., Hilditch, L., et al. (2013). HIV-1 evades innate immune recognition through specific cofactor recruitment. Nature 503, 402-405. doi: 10.1038/nature12769

Rice, G. I., Bond, J., Asipu, A., Brunette, R., Manfield, I. W., Carr, I. M., et al. (2009). Mutations involved in Aicardi-Goutières syndrome implicate SAMHD1 as regulator of the innate immune response. Nat. Genet. 41, 829-832. doi: 10.1038/ng.373

Richards, A., van den Maagdenberg, A. M., Jen, J. C., Kavanagh, D., Bertram, P., Spitzer, D., etal. (2007). C-terminal truncations in human 3'-5' DNA exonuclease TREX1 cause autosomal dominant retinal vasculopathy with cerebral leukodystrophy. Nat. Genet. 39, 1068-1070. doi: 10.1038/ ng2082

Sironi, M., Biasin, M., Forni, D., Cagliani, R., De Luca, M., Saulle, I., et al. (2012). Genetic variability at the TREX1 locus is not associated with natural resistance to HIV-1 infection. AIDS 26, 1443-1445. doi: 10.1097/QAD.0b013e328354b3c2

Stavrou, S., Nitta, T., Kotla, S., Ha, D., Nagashima, K., Rein, A. R., et al. (2013). Murine leukemia virus glycosylated Gag blocks apolipoprotein B editing complex 3 and cytosolic sensor access to the reverse transcription complex. Proc. Nat. Acad. Sci. U.S.A. 110, 9078-9083. doi: 10.1073/pnas. 1217399110

Stetson, D. B., Ko, J. S., Heidmann, T., and Medzhitov, R. (2008). Trex1 prevents cell-intrinsic initiation of autoimmunity. Cell 134, 587-598. doi: 10.1016/j.cell.2008.06.032

Sunseri, N., O'Brien, M., Bhardwaj, N., and Landau, N. R. (2011). HIV-1 modified to package SIV Vpx efficiently infects macrophages and dendritic cells. J. Virol. 85, 6263-6274. doi: 10.1128/JVI.00346-11

Sze, A., Olagnier, D., Lin, R., Van Grevenynghe, J., and Hiscott, J. (2013). SAMHD1 host restriction factor: a link with innate immune sensing of retrovirus infection. J. Mol. Biol. 425, 4981-4994. doi: 10.1016/j.jmb.2013.10.022

Tang, S., Murakami, T., Agresta, B. E., Campbell, S., Freed, E. O., and Levin, J. G. (2001). Human immunodeficiency virus type $1 \mathrm{~N}$-terminal capsid 
mutants that exhibit aberrant core morphology and are blocked in initiation of reverse transcription in infected cells. J. Virol. 75, 9357-9366. doi: 10.1128/JVI.75.19.9357-9366.2001

Unterholzner, L., Keating, S. E., Baran, M., Horan, K. A., Jensen, S. B., Sharma S., et al. (2010). IFI16 is an innate immune sensor for intracellular DNA. Nat. Immunol. 11, 997-1004. doi: 10.1038/ni.1932

Yan, N., Regalado-Magdos, A. D., Stiggelbout, B., Lee-Kirsch, M. A., and Lieberman, J. (2010). The cytosolic exonuclease TREX1 inhibits the innate immune response to human immunodeficiency virus type 1. Nat. Immunol. 11, 1005-1013. doi: 10.1038/ni.1941

Yang, Y., Fricke, T., and Diaz-Griffero, F. (2013). Inhibition of reverse transcriptase activity increases stability of the HIV-1 core. J. Virol. 87, 683-687. doi: 10.1128/JVI.01228-12

Yang, Y., Lindahl, T., and Barnes, D. (2007). Trex1 exonuclease degrades ssDNA to prevent chronic checkpoint activation and autoimmune disease. Cell 131, 873886. doi: 10.1016/j.cell.2007.10.017
Conflict of Interest Statement: The authors declare that the research was conducted in the absence of any commercial or financial relationships that could be construed as a potential conflict of interest.

Received: 14 February 2014; accepted: 11 April 2014; published online: 30 April 2014. Citation: Hasan $M$ and Yan $N$ (2014) Safeguard against DNA sensing: the role of TREX1 in HIV-1 infection and autoimmune diseases. Front. Microbiol. 5:193. doi: 10.3389/fmicb.2014.00193

This article was submitted to Virology, a section of the journal Frontiers in Microbiology. Copyright (C) 2014 Hasan and Yan. This is an open-access article distributed under the terms of the Creative Commons Attribution License (CC BY). The use, distribution or reproduction in other forums is permitted, provided the original author(s) or licensor are credited and that the original publication in this journal is cited, in accordance with accepted academic practice. No use, distribution or reproduction is permitted which does not comply with these terms. 